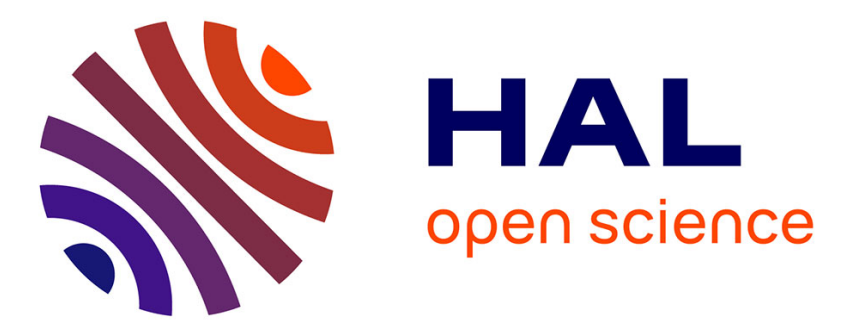

\title{
LA PHOTOLYSE DE LA RIBOFLAVINE DU LAIT. INFLUENCE DE QUELQUES TRAITEMENTS INDUSTRIELS
}

\author{
M. Lhuissier, E. Biette
}

\section{- To cite this version:}

M. Lhuissier, E. Biette. LA PHOTOLYSE DE LA RIBOFLAVINE DU LAIT. INFLUENCE DE QUELQUES TRAITEMENTS INDUSTRIELS. Le Lait, 1964, 44 (439_440), pp.609-613. hal00928366

\section{HAL Id: hal-00928366 https://hal.science/hal-00928366}

Submitted on 1 Jan 1964

HAL is a multi-disciplinary open access archive for the deposit and dissemination of scientific research documents, whether they are published or not. The documents may come from teaching and research institutions in France or abroad, or from public or private research centers.
L'archive ouverte pluridisciplinaire HAL, est destinée au dépôt et à la diffusion de documents scientifiques de niveau recherche, publiés ou non, émanant des établissements d'enseignement et de recherche français ou étrangers, des laboratoires publics ou privés. 


\title{
LA PHOTOLYSE DE LA RIBOFLAVINE DU LAIT. INFLUENCE \\ DE QUELQUES TRAITEMENTS INDUSTRIELS (I)
}

\author{
par \\ M. LHUISSIER et E. BIETTE
}

Laboratoire d'Etude des Qualités biologiques des Aliments de l'Homme, Centre national de Recherches zootechniques, Jouy-en-Josas (S.-O.)

\section{Introduction}

Le lait est une source importante de riboflavine si celui-ci a été protégé de la lumière. Le lait est encore souvent conditionné en bouteilles de verre blanc, et, par suite de précautions insuffisantes, sa teneur en riboflavine peut être abaissée (CAUseret et al., 1961). La riboflavine peut être protégée quand le lait est conditionné en bouteilles de verre ambré (Hugot, LHUissier, Causeret, 1962), ou en emballage opaque - carton imprimé en noir sur sa face interne - (Lhuissier, Hugot, Biette, 1962). Ces divers types de conditionnement étudiés, il importait de savoir si certains traitements subis par le lait ont une influence sur la vitesse de photolyse de la riboflavine.

Stamberg et Theophilds (1945) ont étudié le comportement des laits cru, pasteurisé, ou homogénéisé exposés en bouteilles de verre blanc de "un quart", à la lumière solaire directe, à une température de +16 à $+19^{\circ} \mathrm{C}$.

Les pourcentages de perte de riboflavine observés par ces auteurs ont été les suivants :

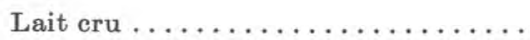

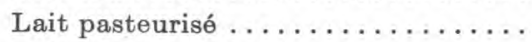

Lait homogénéisé $\ldots \ldots \ldots \ldots \ldots \ldots$

\begin{tabular}{|c|c|}
\hline 1 heure & 2 heures \\
\hline 20 & 40 \\
\hline 20 & 35 \\
\hline 13 & 28 \\
\hline
\end{tabular}

Ziegler (1944) n'a pas mentionné de différence entre le comportement des laits cru, cru homogénéisé, pasteurisé, ou pasteurisé homogénéisé en bouteilles de verre blanc de "un quart ".

Il importait donc de reprendre cette étude de manière systématique en cherchant, non seulement à mettre en évidence d'éven-

(1) Annales de techn. agr. - 1964. Vol. 13, no 1, p. 77. 
tuels effets de la pasteurisation et de l'homogénéisation sur la photolyse de la riboflavine, mais aussi de la préstérilisation et de la stérilisation en bouteilles.

\section{Protocole expérimental}

Les échantillons de lait énumérés sur la figure 1, ont été prélevés le long de la chaîne de traitement d'une usine laitière (à l'exception du lait cru qui a été comparé de façon séparée au lait pasteurisé).

Les traitements appliqués au lait ont été les suivants :

a) Pasteurisation:

- Echantillons des expériences no 1 à 3 : pasteurisation basse : $63-65^{\circ} \mathrm{C}$ pendant 30 minutes ;

- Echantillons des autres expériences : pasteurisation haute : $82^{\circ} \mathrm{C}$ appareil à plaques (1).

b) Préstérilisation :

- Dans un appareil à ultra-haute-température, $141^{\circ} \mathrm{C}$ pendant 5 secondes environ (2);

— Température du lait à la sortie de l'embouteilleuse : + $69^{\circ} \mathrm{C}$

c) Homogénéisation :

- A une pression de $200 \mathrm{~kg}(3)$.

d) Stérilisation en bouteilles:

- Entrée dans le stérilisateur (4) : $66^{\circ} \mathrm{C}$; - sortie du stérilisateur $55-60^{\circ} \mathrm{C}$;

- Durée totale du passage dans le stérilisateur : 51-52 minutes;

- Température maximum et durée de stérilisation : $118,5^{\circ} \mathrm{C}$ pendant 15 minutes.

Chacun des échantillons a été soumis à un éclairement de 3 à 9 minutes par lampe à incandescence (environ 100000 lux au niveau de la surface du lait); le lait étant disposé en couche de $2 \mathrm{~cm}$ d'épaisseur à $24 \mathrm{~cm}$ de la lampe.

Le lait était contenu dans un bécher plongé dans un bain thermostaté. Il a été nécessaire d'installer un dispositif destiné à éviter que la température du lait ne sélève trop fortement : ce dispositif comprenait un soufflage continu d'air comprimé entre la lampe et

(1) Alfa Laval.

(2) Laguilharre, Courbevoie (Seine).

(3) Rammie.

(4) Breil, licence Webster, Paris. 
la surface du lait et un système d'agitation de ce dernier. Dans ces conditions, la température du lait s'est maintenue à $+50^{\circ} \mathrm{C}$ pendant toute la durée de l'irradiation, et la formation d'une " peau " superficielle a été évitée.

La riboflavine a été dosée par voie microbiologique (SNELL, Strong, 1939).

\section{Résultats}

L'étude des résultats reportés sur la figure 1 montre que les traitements étudiés n'ont pas exercé d'influence sensible sur la photolyse de la riboflavine, à l'exception de la stérilisation en bouteilles : dans tous les échantillons de lait stérilisé en bouteilles, la résistance de la vitamine à l'action destructrice de la lumière s'est avérée accrue.

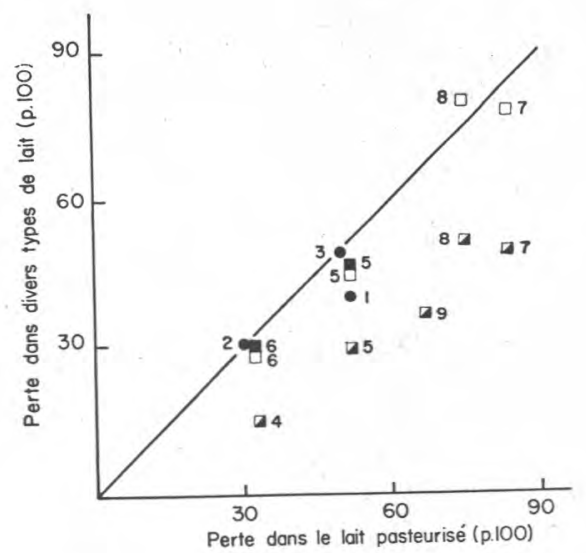

Fig. 1. - Pourcentages de perte observés dans les laits

- cru,

- pasteurisé. préstérilisé,

- pasteurisé, préstérilisé (échantillon 4 et 5), homogénéisé,

- pasteurisé, préstérilisé (échantillons 4 et 5), homogénéisé, stérilisé en bouteilles,

en fonction du pourcentage de perte dans le lait pasteurisé.

Les échantillons portant le même numéro ont même origine et ont été irradiés au cours d'une même expérience.

\section{Discussion}

Nos résultats, contrairement à ceux de STAmberG et Theоpнilus (1945), semblent signifier que l'homogénéisation n'exerce aucun effet sur la résistance de la riboflavine à l'action destructrice de la lumière.

Il faut toutefois remarquer que la teneur du lait en matière grasse peut avoir une forte influence en ce qui concerne l'effet de 
l'homogénéisation sur la transparence du lait aux rayons lumineux, et par contre-coup la vitesse de la photolyse de la riboflavine. Le bris des particules protéiques et surtout des globules gras s'accompagne d'une augmentation de la densité optique du lait (AsнwORTH, 1951).

Mais les mesures effectuées avec une lumière incidente de longueur d'onde voisine de $500 \mathrm{~m} \mu$ correspondant à la destruction maximale de la riboflavine (Josephson, 1946) (0,7 pour le lait pasteurisé, 1,1 pour le lait préstérilisé et pour le lait préstérilisé et homogénéisé, 1,2 pour le lait stérilisé en bouteilles) n'ont pas permis d'établir une relation entre les valeurs de densité optique et la rapidité de la photolyse.

L'effet protecteur entraîné par la stérilisation en bouteilles elle-même ne peut être expliqué actuellement. On peut se demander si, du fait du traitement thermique relativement sévère qu'implique la stérilisation en bouteilles, la riboflavine passe sous une forme moins sensible à l'action de la lumière, ou bien s'il y a disparition de facteurs qui favorisent la photolyse de la riboflavine ou encore apparition de facteurs protecteurs.

\section{Remerciements}

Nous adressons nos vifs remerciements à MHe BeJAMBES, Directeur de Recherches à l'I.N.R.A., à M. VEnET, Directeur des Gares laitières de la Région parisienne (Société Laitière Métropolitaine) ainsi qu'à M. Dulex, Directeur de l'usine d'Ivry (S.L.M.) et M. LAROQUE, chimiste (S.L.M.), grâce à qui nous avons pu nous procurer les échantillons nécessaires à cette étude.

\section{Summary}

Riboflavin photolysis has been studied in various types of milk:

I raw,

II pasteurized,

III pasteurized, presterilized (UHT),

IV pasteurized, presterilized, homogenized,

$V$ pasteurized, presterilized, homogenized, sterilized in-bottle.

The loss of riboflavin in these products after exposure to artificial light was the same in all cases except for milk sterilized in bottle, where it was lower. The reason for the protective effect exercised by this sterilization was not understood.

\section{RÉFÉRENCES BIBLIOGRAPHIQUES}

AshwORTH U. S. 1951. Turbidity as a means for determining the efficiency of homogenization. J. Dairy Sci., 34 (4), 317-320. 
Causeret J., Hugot D., Goulas-Scholler C., Mocquot G., 1961. Evolution de la teneur du lait stérilisé en riboflavine et en vitamine A au cours d'une conservation de longue durée. Ann. Techn. agric., 10, 289-300.

Hugot D., Lhuissier M., Causeret J., 1962. Effet protecteur des bouteilles de verre ambré contre les pertes de riboflavine et de vitamine $\mathrm{A}$ dues à l'exposition du lait stérilisé à la lumière. Ann. Techn. agric., 11, 145-151.

JosEPHSON D. V., 1946. Some observations regarding the effect of various wavelenghts of light on the riboflavin content and flavor of milk. $J$. Dairy Sci., 29, 508-510.

Lhuissier M., Hugot D., Biette E., 1962. Etude vitaminologique du lait "upérisé " conditionné aseptiquement en récipients Tetra Pak : influence de la conservation. L'Industrie laitière, 194, 319-320.

Snell E. E., Strong F. M., 1939. A microbiological assay for riboflavin. Industri. Engng. Chem. Anal. Ed., 11, 346.

Stamberg O. E., Theophilus D. R., 1945. Photolysis of riboflavin in milk. J. Dairy Sci., 28, 269.

ZIEGLER J. A., 1944. Photochemical destruction of vitamine $\mathrm{B}_{2}$ in milk. J. Amer. Chem. Soc., 66, 1039.

\title{
L'UTILISATION DE COLORANTS POUR DÉCELER LA PRÉSENCE D'ANTIBIOTIQUES DANS LE LAIT (1)
} (fin)

\author{
par \\ Professeur Docteur P. KästLI (Suisse)
}

\subsection{Essais effectués par nos soins [24].}

Afin de pouvoir former notre propre opinion sur les effets produits par les additions de colorants, nous avons procédé dans notre Institut à quelques essais qui devront être étendus par la suite.

\subsubsection{Essais au moyen de chlorophylle.}

Deux vaches ont été traitées en même temps chacune sur deux quartiers à l'aide d'une préparation contenant $100000 \mathrm{U}$ de pénicilline $\mathrm{G}$ sous forme de sel de potassium, 0,4 g d'une solution huileuse de chlorophylle, ainsi que de l'oleum sésame et du stéarate d'aluminium pour $10 \mathrm{ml}$. On a obtenu les résultats suivants :

(1) Bulletin F.I.L. 1963. VI (Rapport présenté par le $\mathrm{P}^{\mathrm{r}} \mathrm{D}^{\mathrm{r}} P$. Kästli et reproduit avec l'autorisation de la Fédération Internationale de Laiterie). 